\title{
Order within associations as a test of association-memory models
}

\author{
Jeremy B Caplan ${ }^{1 *}$, Mayank Rehani ${ }^{2}$ \\ From Nineteenth Annual Computational Neuroscience Meeting: CNS*2010 \\ San Antonio, TX, USA. 24-30 July 2010
}

In learning associations (e.g., a pairing of items, A-B), the hippocampus appears to implement Associative Symmetry, namely, when learning a forward association $(\mathrm{A}->\mathrm{B})$, picking up the backward association $(\mathrm{B}->\mathrm{A})$ for free [3], a characteristic of human association-memory that has been replicated numerous times (e.g., [5]). A mathematical operation that does this automatically, and thus might be carried out by the hippocampus, is the convolution operation, the operation used to store associations in a range of influential behavioural memory models [2]. Convolution-based models lead to a specific prediction about within-pair order memory (the participant's ability to retrieve the relative orders of the A and $B$ items), namely, that within-pair order memory should be at chance levels. In contrast, models based on the outer product, known as matrix models [1] the way they have been applied, lead to perfect within-pair order memory (assuming the pairing is retrieved); likewise for numerous other models that assume associations are stored by concatenating the vector representations of paired items [6].

Here we test within-pair order memory with a verbal double-function list paradigm in which participants are presented with pairs of words in which the left-handed item of one pair is the right-handed item of a different pair. Thus, within-pair order information is critical for later effective cued recall. The results suggested that human participants have neither poor nor near-perfect memory for within-pair order, challenging all current models to our knowledge. Our recently proposed positional coding model for paired-associate memory [4], which already incorporates within-pair order in the same manner as between-pair order. Even this positional coding model requires some additional assumptions to fit the fine structure of the behavioural data.

In sum, our findings suggest that within-pair order memory is neither poor nor perfect, pointing to a fallible mechanism for within-pair order learning in verbal association memory tasks and constraining the computational mechanisms the hippocampus could plausibly use to learn pairs with the property of Associative Symmetry.

\section{Author details}

${ }^{1}$ Psychology Department and Centre for Neuroscience, University of Alberta, Edmonton, AB, T6G 2E9, Canada. 'Department of Rehabilitation Science, University of Alberta, Edmonton, AB, T6G 2G4, Canada.

\section{Published: 20 July 2010}

\section{References}

1. Anderson JA: Two models for memory organization using interacting traces. Mathematical Biosciences 1970, 8(z):137-160.

2. Borsellino A, Poggio T: Holographic aspects of temporal memory and optomotor responses. Kybernetik 1972, 10:58-60.

3. Bunsey M, Eichenbaum HB: Conservation of hippocampal memory function in rats and humans. Nature 1996, 379:255-257.

4. Caplan JB: Associative Isolation: unifying associative and order paradigms. Journal of Mathematical Psychology 2005, 49:383-402.

5. Kahana MJ: Associative symmetry and memory theory. Memory \& Cognition 2002, 30:823-840.

6. Mensink G, Raaijmakers JGW: A model for interference and forgetting. Psychological Review 1988, 95:434-455.

doi:10.1186/1471-2202-11-S1-P78

Cite this article as: Caplan and Rehani: Order within associations as a test of association-memory models. BMC Neuroscience 201011 (Suppl 1): P78.

\footnotetext{
* Correspondence: jcaplan@ualberta.ca

${ }^{1}$ Psychology Department and Centre for Neuroscience, University of Alberta, Edmonton, AB, T6G 2E9, Canada
} 